\title{
On some convexity properties of Orlicz spaces of vector valued functions
}

\author{
H. HUDZIK
}

\begin{abstract}
A "stability" theorem that is a generalization of Th. 6 in [2] for the modulus of convexity of Banach spaces is given. Necessary and sufficient conditions for $\delta_{L} \Phi(a)>0$, where $a \in(0,2]$, in Orlicz spaces $L^{\Phi}(\mu, X)$ of vector valued functions are given. The convexity coefficient $\varepsilon_{0}\left(L^{\Phi}(\mu, X)\right)$ is computed for these spaces. The equality $\varepsilon_{0}\left(L^{\Phi}(\mu, X)\right)=\varepsilon_{0}(X)$ for Orlicz-Bochner spaces generated by uniformly convex Orlicz functions satisfying the $\Delta_{2}$-condition is showed.
\end{abstract}

\section{INTRODUCTION}

Throughout this paper $(T, \Sigma, \mu)$ denotes a non-atomic, infinite and complete measure space and $X$ denotes a Banach space. A function $\Phi: X \rightarrow[0,+\infty]$ is said to be an Orlicz function if it is convex, even, lower semicontinuous, vanishing and continuous at zero, and $\Phi \neq 0, F(T, X)$ stands for the space of all equivalence classes of strongly $\Sigma$-measurable functions from $T$ into $X$.

Given an Orlicz function $\Phi$, we define the Orlicz space $L^{\Phi}(\mu, X)$ as the set of all functions $f \in F(T, X)$ such that

$$
I_{6}(\lambda f)=\int_{T} \Phi(\lambda f(t)) \mathrm{d} \mu<+\infty
$$

for some $\lambda>0$ depending on $f$. This space equipped with the Luxemburg norm

$$
\|f\|_{\Phi}=\inf \left\{\lambda>0: I_{\Phi}(\lambda-1 f) \leq 1\right\}
$$

is a normed space (see [11-13]), and it is a Banach space if and only if $\Phi$ is uniformly large at infinity, i.e.

$\lim \inf \{\Phi(x):\|x\|=k\}=+\infty$ (see [15]).

$k \rightarrow+\infty$

1980 Mathematics Subject Classification (1985 revision): 46E30

Editorial de la Universidad Complutense. Madrid, 1989. 
We say an Orlicz function $\Phi$ satisfies the $\Delta_{2}$-condition if there is a constant $K>0$ such that $\Phi(2 x) \leq K \Phi(x)$ for all $x \in X$.

The modulus of convexity of a normed space $(X, \|)$ is the function $\delta_{x}(\cdot):(0,2] \rightarrow[0,1]$ defined by

$$
\delta_{x}(\varepsilon)=\inf \{1-\|1 / 2(x+y)\|:\|x\| \leq 1,\|y\| \leq 1,\|x-y\| \geq \varepsilon\} .
$$

The convexity coeffcient of a normed space $(X,\|\|)$ is defined by

$$
\varepsilon_{0}(X)=\sup \left\{\varepsilon \in[0,2]: \delta_{X}(\varepsilon)=0\right\}(\sup \text { ø } \stackrel{\operatorname{def}}{=} o) \text {. }
$$

(see [2]).

\section{RESULTS}

To prove the first theorem we shall need the following

Lemma 1. If $\delta_{Y}(a)>0$ for a number $a \in(0,2)$, then there is a number $\gamma>1 /$ a such that $\operatorname{a\gamma }\left(1-\delta_{x}(1 / \gamma)\right)=1$.

Proof. By the assumptions and by the continuity of $\delta_{x}$ it follows that there is a number $\alpha>1 / a$ such that $\delta_{x}(1 / \alpha)>0$. So, $a \beta\left(1-\delta_{x}(1 / \alpha)\right)<1$ for a certain $\beta>1 / a$. Taking $\alpha_{0}=\min (\alpha, \beta)$, we have $a \alpha_{o}\left(1-\delta_{x}\left(1 / \alpha_{0}\right)\right)<1$.

A function $h:(1 / 2,+\infty) \rightarrow R_{+}$defined by $h(\lambda)=a \lambda\left(1-\delta_{x}(1 / \lambda)\right)$ is continuous and $h(\lambda) \rightarrow+\infty$ as $\lambda \rightarrow+\infty$. Since $h\left(\alpha_{o}\right)<1$, the Darboux property of $h$ yields $h(\gamma)=a \gamma\left(1-\delta_{X}(1 / \gamma)\right)=1$ for a certain $\gamma>1 / a$ which finishes the proof.

Now, we are able to generalize Th. 6 in [2]. The proof is almost the same but we shall give it for the sake of completness.

Theorem 2. Let $X$ be a Banach space with $\varepsilon_{o}(X)$ in the interval $[0, a)$, where $0<a \leq 2$. Let $\gamma>1 / a$ be such that $a \gamma\left(1-\delta_{x}(1 / \gamma)\right)=1$. If $Y$ is a Banach space with Banach-Mazur distance $d(X, Y)<a \gamma$, then $\varepsilon_{0}(Y)<a$.

Proof. Without loss of generality we may assume that $U$ is an isomorphism between $X$ and $Y$ such that $\left\|U^{-1}\right\|=1$ and $\mathrm{d}(X, Y) \leq\|U\| \leq a b \gamma$, where $0<b<1$. Let $y_{1}, y_{2} \in S_{Y}(=$ the unit sphere of $Y),\left\|y_{1}-y_{2}\right\| \geq\|U\| / \gamma$ and $x_{1}=U^{-1} y_{1}$, $x_{2}=U^{-1} y_{2}$. Then $\left\|x_{1}\right\| \leq 1,\left\|x_{2}\right\| \leq 1$ and $\|U\| / \gamma \leq\left\|y_{1}-y_{2}\right\|=$ $\left\|U\left(x_{1}-x_{2}\right)\right\| \leq\|U\|\left\|x_{1}-x_{2}\right\|$, whence $\left\|x_{1}-x_{2}\right\| \geq 1 / \gamma$. Since $a \gamma>1$, by the equality $a \gamma\left(1-\delta_{x}(1 / \gamma)\right)=1$, it follows that $\delta_{x}(1 / \gamma)>0$. Therefore

$$
\left\|\left(y_{1}+y_{2}\right) / 2\right\|=\left\|U\left(1 / 2\left(x_{1}+x_{2}\right)\right)\right\| \leq\|U\|\left\|1 / 2\left(x_{1}+x_{2}\right)\right\| \leq a b \gamma\left(1-\delta_{X}(1 / \gamma)\right)=b_{0}
$$

This means that $\delta_{\gamma}(\|U\| / \gamma) \geq 1-b>0$. Thus, $\varepsilon_{o}(Y) \leq\|U\| / \gamma<a$. 
In the fixed point theory the notion of the convexity coefficient is useful (see [2]). We shall give now a basic theorem to compute $\varepsilon_{o}\left(L^{\Phi}(\mu, X)\right)$.

Theorem 3. Let a be a number in $(0,2]$. The following conditions are equivalent:

$1^{\circ} \delta_{L} \propto(a)>0$.

$2^{\circ}$ (a) there is $\delta \in(0,1)$ such that for every $x, y \in X$ satisfying the equality $\Phi((x-y) / a) \geq(1-\delta) \Phi((x+y) / 2)$, we have $\Phi((x+y) / 2) \leq \frac{1-\delta}{2}\{\Phi(x)+\Phi(y)\}$,

(b) $\Phi$ satisfies the $\Delta_{2}$-condition.

Proof. $2^{\circ} \Rightarrow 1^{\circ}$. Assume that $\|f\|_{\Phi} \leq 1,\|g\|_{\Phi} \leq 1$ and $\|f-g\|_{\Phi} \geqslant a$. Then $I_{\phi}(f) \leq 1, I_{\phi}(g) \leq 1$ and $I_{\Phi}((f-g) / a) \geq 1$. Define

$$
A=\{t \in T: \Phi((f(t)-g(t)) / a) \geq(1-\delta) \Phi((f(t)+g(t)) / 2)\} .
$$

Then

$$
I_{\Phi}\left(\frac{f-g}{a} \chi_{T M}\right) \leq \frac{1-\delta}{2}\left\{I_{\Phi}\left(f \chi_{M}\right)+\left(I_{\odot}\left(g \chi_{T M}\right)\right\} \leq 1-\delta\right.
$$

Consequently, $I_{\Phi}\left(\frac{f-g}{a} \chi_{A}\right) \geq \delta$. By the $\Delta_{2}$-condition, we get

$$
I_{\Phi}\left(\frac{f-g}{a} \chi_{\phi}\right) \leq K\left\{I_{\Phi}\left(f \chi_{1}\right)+I_{\Phi}\left(g \chi_{\oplus}\right)\right\}
$$

where $K$ is a constant depending only on $a$ and $\Phi$. Hence,

$$
\begin{aligned}
1-I_{\Phi}\left(\frac{f+g}{2}\right) & \geq(1 / 2)\left\{I_{\Phi}(f)+I_{\Phi}(g)\right\}-I_{\Phi}\left(\frac{f+g}{2}\right) \\
& \geq(1 / 2)\left\{I_{\Phi}\left(f \chi_{1}\right)+I_{\Phi}\left(g \chi_{A}\right)\right\}-I_{\Phi}\left(\frac{f+g}{2} \chi_{1}\right) \\
& \geq(1 / 2)\left\{I_{\Phi}\left(f \chi_{A}\right)+I_{\Phi}\left(g \chi_{1}\right)\right\}-\frac{1-\delta}{2}\left\{I_{\Phi}\left(f \chi_{A}\right)+I_{\Phi}\left(g \chi_{A}\right)\right\} \\
& =(\delta / 2)\left\{I_{\Phi}\left(f \chi_{1}\right)+I_{\Phi}\left(g \chi_{1}\right)\right\} \geq \frac{\delta^{2}}{2 K}
\end{aligned}
$$


Equivalently,

$$
I_{\Phi}\left(\frac{f+g}{2}\right) \leq 1-\frac{\delta^{2}}{2 K}
$$

Applying the $\triangle_{2}$-condition, we get

$$
\left\|\frac{f+g}{2}\right\|_{\Phi} \leq 1-p\left(\frac{\delta^{2}}{2 K}\right),
$$

where $p$ is a function from $(0,1)$ into itself (in the real case see [4] and [6]).

This yields $\delta_{L} \leftrightarrow(a) \geq p\left(\frac{\delta^{2}}{2 K}\right)>0$.

$1^{\circ} \Rightarrow 2^{\circ}$. If $\Phi$ does not satisfy the $\Delta_{2}$-condition, then $L^{\circ}(\mu, X)$ contains an isometric copy of $l^{\infty}$ (see [3] and [4]). Therefore, $\delta_{L} \alpha(a) \leq \delta_{\rho \circ}(a)=0$ for every $a \in(0,2]$. Assume now that condition $2^{\circ}(a)$ is not satisfied, i.e. for every $\delta \in(0,1)$ there exist $x, y \in X$ such that

$$
\Phi(1 / a(x-y)) \geq(1-\delta) \Phi(x+y) / 2) \text { and } \Phi((x+y) / 2)>1 / 2(1-\delta)\{\Phi(x)+\Phi(y)\} .
$$

Let $B, C \in \sum, B \subset C$, be such that $\mu(C)=\mu(B \backslash C)$ and $(\Phi(x)+\Phi(y)) \mu(B)=2$.

Define

$$
f=x \chi_{C}+y \chi_{B, C} g=y \chi_{C}+x \chi_{B C}
$$

We have $I_{\Phi}(f)=I_{\Phi}(g)=1$, whence $\|f\|_{\Phi}=\|g\|_{\Phi}=1$. Moreover,

$$
\begin{aligned}
& I_{\Phi}\left((f-g) /(1-\delta)^{2} a\right) \geq\left(1 /(1-\delta)^{2}\right) \int_{B} \Phi((x-y) / a) d \mu \\
& \geq\left(1 /(1-\delta)^{2}\right) \Phi((x-y) / a) \mu(B) \geq(1 /(1-\delta)) \Phi((x+y) / 2) \mu(B) \\
& \geq(1 / 2)\{\Phi(x)+\Phi(y)\} \mu(B)=1
\end{aligned}
$$

Therefore $\|(f-g) / a\|_{\Phi} \geq(1-\delta)^{2}$. In an analogous way the inequality $\|(f+g) / 2\|_{\Phi} \geq 1-\delta$ car be proved. Since $\delta \in(0,1)$ was arbitrary, this means that $\delta_{L} \leftrightarrow(a)=0$. The theorer 1 is proved.

To prove the next theorem, we will need the following

Proposition 4. Li $\Phi$ be an Orlicz function satisfying the $\Delta_{2}$-condition. Then the following ass ertions are equivalent: 
On some convexity properties of Orlicz spaces of vector...

(+) there is $\delta \in(0,1)$ such that $\Phi((x+y) / 2 \leq((1-\delta) / 2)\{\Phi(x)+\Phi(y)\}$ whenever $x, y \in X$ satisfy $\Phi((x-y) / a) \geq(1-\delta) \Phi((x+y) / 2)$.

$(++)$ there is $\sigma \in(0,1)$ such that $\Phi((x+y) / 2) \leq((1-\sigma) / 2)\{\Phi(x)+\Phi(y)\}$ whenever $x, y \in X$ satisfy $\Phi((x-y) / a(1-\sigma)) \geq \Phi((x+y) / 2)$.

Proof. $(++) \Rightarrow(+)$. Assume that $\Phi((x-y) / a) \geq(1-\sigma) \Phi((x+y) / 2)$. Then $\Phi((x-y) / a(1-\sigma)) \geq(1 /(1-\sigma)) \Phi((x-y) / a) \geq \Phi((x+y) / 2)$. In view of condition $(++)$, we have $\Phi((x+y) / 2) \leq((1-\sigma) / 2)\{\Phi(x)+\Phi(y)\}$.

Thus, it suffices to put $\delta=\sigma$.

$(+) \Longrightarrow(++)$. Assume that $\Phi\left((x-y) / a\left(1-\sigma_{1}\right)\right) \geq \Phi((x+y) / 2)$, where $\sigma_{1}$ is a constant in $(0,1)$ satisfying $\Phi\left(x /\left(1-\sigma_{1}\right)\right) \leq(1 /(1-\delta)) \Phi(x)$ for every $x \in X$ (by the $\Delta_{2}$-condition such a constant exists) and $\delta$ is the constant from condition $(+)$. Then

$$
\Phi((x-y) / a)(1 /(1-\delta)) \geq \Phi\left((x-y) / a\left(1-\sigma_{1}\right)\right) \geq \Phi((x+y) / 2) .
$$

Therefore, by condition $(+)$, we get

$$
\Phi((x+y) / 2) \leq((1-\delta) / 2)\{\Phi(x)+\Phi(y)\} .
$$

It suffices to put $\sigma=\min \left(\sigma_{1}, \delta\right)$.

Theorem 5. Let $\Phi$ be a uniformly convex Orlicz function defined on the real line, i.e. for every $a \in(0,1)$ there exists $\delta(a) \in(0,1)$ such that for every $u \in R$ we have $\Phi((u+a u) / 2) \leq(1 / 2)(1-\delta(a))\{\Phi(u)+\Phi(a u)\}$, and let $\Phi$ satisfy the $\Delta_{2}$-condition and $(X,\|\|)$ be a Banach space. Then $\delta_{L} \leftrightarrow(\varepsilon)>0$ for the Orlicz-Bochner space $L^{\oplus}=L^{\oplus}(\mu, X)$ if and only if $\delta_{x}(\varepsilon)>0$.

Proof. Since $X$ can be isometrically embedded into $L^{\oplus}(\mu, X)$, the condition $\delta_{X}(\varepsilon)=0$ implies $\delta_{L} \leftrightarrow(\varepsilon)=0$.

Now, in view of Proposition 4 assume that $\delta_{x}(\varepsilon)>0$. It suffices to show that there exists a constant $\sigma \in(0,1)$ such that for every $x, y \in X$, we have

(1) $\left\|\frac{x-y}{\varepsilon \sigma}\right\| \geq\left\|\frac{x+y}{2}\right\|$ implies $\Phi\left(\left\|\frac{x+y}{2}\right\|\right) \leq \frac{\sigma}{2}\{\Phi(\|x\|)+\Phi(\|y\|)\}$.

Since $\delta_{X}(\varepsilon)>0$ by the assumption, there exists $\delta \in(0,1)$ such that

(2) $\left\|\frac{x+y}{2}\right\| \leq \delta$ whenever $x, y \in B_{x}$ (= the unit ball of $X$ ) and

$$
\left\|\frac{x-y}{\varepsilon \delta}\right\| \geq\left\|\frac{x+y}{2}\right\| \text {. }
$$


Assume that $x, y \in X$ and $\left\|\frac{x-y}{\varepsilon \delta}\right\| \geq\left\|\frac{x+y}{2}\right\|$. We can assume without loss of generality that $\|x\| \leq\|y\|$. Then $\frac{x}{\|y\|}, \frac{y}{\|y\|} \in B_{x}$ and

$\left\|\frac{x-y}{\varepsilon \delta\|y\|}\right\| \geq\left\|\frac{x+y}{2\|y\|}\right\|$. Thus, in virtue of condition (2), we get $\left\|\frac{x+y}{2}\right\| \leq \delta\|y\|$. Now, we shall consider two cases.

$1^{\circ} \cdot \sqrt{\delta}\|y\| \leq\|x\|$. Then

$$
\begin{gathered}
\left\|\frac{x+y}{2}\right\| \leq \delta\|y\|=\delta \frac{\|y\|+\|y\|}{2} \leq \delta \frac{\|x\| / \sqrt{\delta}+\|y\|}{2} \leq \delta \frac{\|x\|+\|y\|}{2 \sqrt{\delta}} \\
=\frac{\sqrt{\delta}}{2}(\|x\|+\|y\|) .
\end{gathered}
$$

Hence

$$
\Phi\left(\left\|\frac{x+y}{2}\right\|\right) \leq \frac{\sqrt{ } \delta}{2}\{\Phi(\|x\|)+\Phi(\|y\|)\} .
$$

$2^{\circ}$. $\|x\|<\sqrt{\delta}\|y\|$. By uniform convexity of $\Phi$, we have

$$
\Phi\left(\left\|\frac{x+y}{2}\right\|\right) \leq \Phi\left(\frac{\|x\|+\|y\|}{2}\right) \leq \frac{1-\eta(\delta)}{2}\{\Phi(\|x\|)+\Phi(\|y\|)\} .
$$

Therefore, for every $x, y \in X$ such that $\left\|\frac{x-y}{a \delta}\right\| \geq\left\|\frac{x+y}{2}\right\|$, we have

$$
\Phi\left(\left\|\frac{x+y}{2}\right\|\right) \leq \frac{\max ((1-\eta(\delta)), \sqrt{ } \delta)}{2}\{\Phi(\|x\|)+\Phi(\|y\|)\} .
$$

To prove condition (1) it suffices to put $\sigma=\max ((1-\eta(\delta), \sqrt{\delta})$.

Note. The thesis of Theorem 5 means that $\varepsilon_{0}\left(L^{\oplus}(\mu, X)\right)=\varepsilon_{o}(x)$. It is a generalization of Theorem 9 of Downing and Turett [2]. However, our method of the proof is quite different than the method used there.

An Orlicz function $\Phi$ is said to satisfy condition $C_{a}(a \in(0,2))$ if there exists a number $\sigma \in(0,1)$ such that

$$
\Phi((x+y) / 2) \leq(\sigma / 2)\{\Phi(x)+\Phi(y)\}
$$


whenever $x, y \in X$ and $\Phi((x-y) / a \sigma) \geq \Phi((x+y) / 2)$.

For any Orlicz function $\Phi$ we define the parameter

$$
\alpha(\Phi)=\inf \left\{a \in(0,2): \Phi \text { satisfies condition } C_{s}\right\} .
$$

We shall give now an inmediate consequence of Th. 3 and Prop. 4.

Corollary 6. Let $\Phi$ be an Orlicz function. Then $\varepsilon_{o}\left(L^{\Phi}(\mu, X)\right)=2$ whenever $\Phi$ does not satisfy the $\Delta_{2}$-condition and $\varepsilon_{0}\left(L^{\Phi}(\mu, X)\right)=\alpha(\Phi)$ in the opposite case.

Note. Theorem 3 and Corollary 6 generalize the results of [6] to Orlicz spaces of vector valued functions. Theorem 3 generalizes also some results of [4], [5] and [7]. These results are also connected with the results of [8], [9] and $[10]$.

Corollary 7. Let $\Phi$ be an Orlicz function defined on the real line $R$ and $(X,\|\|)$ be a Banach space. Then the Orlicz-Bochner space $L^{\oplus}(\mu, X)$ is uniformly rotund if and only if both spaces $L \Phi(\mu, R)$ and $X$ are uniformly rotund.

Recall that $L^{\Phi}(\mu, R)$ is uniformly rotund if and only if $\Phi$ is uniformly convex and satisifes the $\triangle_{2}$-condition (see [7]).

Problem. Is the equality $\varepsilon_{o}\left(L^{\bullet}(\mu, X)\right)=\max \left(\varepsilon_{o}\left(L^{\bullet}(\mu, R), \varepsilon_{o}(X)\right)\right.$ true for every Orlicz-Bochner space?

Added in proof. The problem has negative answer. We refer to the paper of the author and T. Landes entitled "Characteristic of convexity of Köthe function spaces" (preprint).

\section{References}

[1] S. CHEN and H. HUDZIK, On some convexities of Orlicz and Orlicz-Bochner spaces, Comment. Math. Univ. Carol. 29, No.1(1988), 13-29.

[2] D. J. DowING and B. TURETT, Some properties of the characteristic of convexity relating to fixed point theory, Pacific J. Math. 104, No. 2 (1983), 343-350.

[3] H. HuDzIK, On some equivalent conditions in Musielak-Orlicz spaces, Comment. Math. 24 (1984), 57-64.

[4] H. HUDzIK, Some class of uniformly non-square Orlicz-Bochner spaces, Comment. Math. Univ. Carol. 26, No.2(1985), 269-274. 
[5] H. HudizK, Convexity in Musielak-Orlicz spaces, Hokkaido Math. J. 14(1985), 85-96.

[6] H. HUdZIK, A. KAMINSKA and J. MUSIELAK, On the convexity coefficient of Orlicz spaces, Math. Zeit. 197 (1988), 291-295.

[7] A. KaminSKA, On uniform convexity of Orlicz spaces, Indag. Math. 44 (1982), 27-36.

[8] A. KAMINSKA and B. TURETT, Uniformly non- $l_{n}^{(n)}$ Orlicz-Bochner spaces, Bull. Acad. Polon. Sci. Math. 35, No. 3-4 (1987), 211-218.

[9] M. A. KHAMSI, W.M. KozLOWSKI and CH. SHUTAO, Some geometrical properties and fixed point theorems in modular spaces (to appear).

[10] M. A. KHAMSI, W.M. KozLowSKI and S. REICH, Fixed point theory in modular function spaces (to appear).

[11] M. A. KRASNOSELSKII and YA. B. RUTICKII, Convex functions and Orlicz spaces, Groningen 1961 (translation).

[12] W. A. J. LuXEMBURG, Banach function spaces, Thesis, Delft 1955.

[13] J. MUSIELAK, Orlicz spaces and modular spaces, Lectures Notes in Math. 1034, Springer-Verlag, Berlin, Heidelberg, New York, Tokyo (1983).

[14] B. TURETT, Rotundity of Orlicz spaces, Indag. Math. 38 (1976), 462-469.

[15] B. TuretT, Fenchel Orlicz spaces, Dissertat. Math. 131 (1980), 1-60.

Institute of Mathematics

A. Mickiewicz University and Mathematical Institute of the Polish Academy of Sciences (Poznan Branch)

Poznan, Poland 